\title{
Horizontal transfer of a non-autonomous Helitron among insect and viral genomes
}

Brad S Coates ${ }^{1,2}$

\begin{abstract}
Background: The movement of mobile elements among species by horizontal transposon transfer $(H T T)$ influences the evolution of genomes through the modification of structure and function. Helitrons are a relatively new lineage of DNA-based (class II) transposable elements (TES) that propagate by rolling-circle replication, and are capable of acquiring host DNA. The rapid spread of Helitrons among animal lineages by HTT is facilitated by shuttling in viral particles or by unknown mechanisms mediated by close organism associations (e.g. between hosts and parasites).

Results: A non-autonomous Helitron independently annotated as BmHel-2 from Bombyx mori and the MITE01 element from Ostrinia nubilalis was predicted in the genomes of 24 species in the insect Order Lepidoptera. Integrated Helitrons retained $\geq 65 \%$ sequence identity over a 250 bp consensus, and were predicted to retain secondary structures inclusive of a $3^{\prime}$-hairpin and a $5^{\prime}$-subterminal inverted repeat. Highly similar Hel-2 copies were predicted in the genomes of insects and associated viruses, which along with a previous documented case of real-time virus-insect cell line transposition suggests that this Helitron has likely propagated by HTT.

Conclusions: These findings provide evidence that insect virus may mediate the HTT of Helitron-like TEs. This movement may facilitate the shuttling of DNA elements among insect genomes. Further sampling is required to determine the putative role of HTT in insect genome evolution.
\end{abstract}

Keywords: Horizontal transposon transfer, Viral vectors

\section{Background}

Helitrons are class II DNA transposable elements (TEs) that were initially discovered in the Arabidopsis thaliana genome and have subsequently been described in a number of diverse eukaryotic genomes where they occupy 1.3 to $6.6 \%$ of the total nucleic acid content [1-5]. In contrast to the cut-and-paste propagation of other class II TEs, Helitrons transpose in a conservative fashion where original integrations are retained due to the synthesis of daughter TEs by rolling circle replication (RCR) [1]. This type of replication is similar to that used by ssDNA viruses, bacteria, and plasmids, and involves the enzymatic activity of Replicase/Helicase (RepHel) proteins. Eukaryotic RepHels show strong homology with Rep domain-containing proteins encoded by ssDNA geminiviruses such that they are presumed to have been acquired

\footnotetext{
Correspondence: brad.coates@ars.usda.gov

'United States Department of Agriculture, Agricultural Research Service, Corn Insects \& Crop Genetics Research Unit, lowa State University, Ames, IA 50011, USA

${ }^{2}$ Department of Entomology, lowa State University, Ames, IA 50011, USA
}

by HTT [6], and mediate RCR of autonomous as well as non-autonomous Helitron elements in the same genome. Nascent Helitron copies integrate at novel genome locations between AT or TT di-nucleotides and do not produce target site duplications. The integration of Helitrons in plant genomes are believed to involve the transposase, RPA-TPase, which shares homology to the largest subunit of the replication protein A (RPA70). Accumulating evidence indicates that Helitron integrations can have functional consequences resulting from gene knockouts that lead to observable phenotypic variants $[7,8]$, or insertion mutations that change the genetic architecture and the expression of affected genes [9]. Moreover, Helitrons have been implicated in exon shuffling [10], transcription readthrough [11], and the mobilization of acquired host genome regions $[5,12,13]$.

Helitron integrations in plant genomes can be highly cryptic and difficult to predict outside of conserved 5' -TC and $3^{\prime}$-CTRR termini, and a stem-loop structure near the 3 '-terminus [12,14]. In many instances, the comparison of haplotype variants in a species or sophisticated computer 
algorithms are relied upon to predict the myriad of variant Helitron integrations in or among genomes. In contrast, Helitron sequences show high levels of intraspecific conservation among integrations in Drosophila species [4], the little brown bat, Myotis lucifugus [15] and among multiple species in the insect Order Lepidoptera $[5,13,16,17]$. Additionally, conserved secondary structures are predicted among Helitrons from Lepidoptera and Diptera, which form two inverted repeats [a $5^{\prime}$-inverted repeat ( $5^{\prime}$-IR), and base pairing between subterminal inverted repeats (SIRs)] that may be required for replication, mobilization, or integration $[4,13,18]$. This dramatic sequence and structural conservation among Helitrons in the genomes of divergent insect species was surmised by some to be a consequence of a rapid burst in transposition within and among genomes that has been facilitated by HTT. Specifically, horizontal transfer broadly refers to the exchange of genetic material between established lineages and has been detected for transposons, group I introns, and entire gene sequences $[18,19]$, yet details on the exact mechanism of HTT remain somewhat elusive. Endoparasitic and endosymbiotic organisms can acquire segments of host DNA by intermolecular recombination [20] or following the integration of host-derived mobile genetic elements [21]. The infection of multiple host species by these pathogens has been implicated as a mechanism that vectors DNA across species boundaries. In a recent study, the rapid proliferation of two Helitrons, Heligloria and Helisimi, among insect species were predicted to involve HTT mediated by the dsDNA Cotesia plutella and C. sesamiae bracoviruses [17]. The C. plutella and C. sesamiae bracoviruses are aptly named due to the respective infection of the hymenopteran wasps C. plutella and C. sesamiae that are in-turn ectoparasitoids of several stem boring larval Lepidoptera including Plutella xylostella [22], Sesamia calamistis, Busseola fusca, Chilo partellus, and Chilo orichalcociliellus [23]. A Helitron with high shared sequence identity in the genome of a virus and insect host species provided comparative evidence that these mobile DNA elements are moving between genomes, and that HTT is likely a mechanism by which Helitrons have colonized the genomes of related insects [17]. Furthermore, this evidence agreed with prior finding that TEs can be shuttled among genomes by virus- and parasite-host insect interactions [24,25].

Despite this evidence, the evolutionary scope or the methods by which HTT occur remains unclear. For instance, the HTT of DNA transposons between the genomes of animal hosts and their parasitic insects was recently detected, where it was hypothesized that intracellular parasites, viruses, or extrachromosomal material might vector TE movements between genomes of different species, such as between the blood-feeding hexapod Rhodnius prolixus and tetrapods [26]. This evidence suggested that HTT of genetic material even among highly divergent species may be more prevalent than previously envisioned. Research herein provides evidence that a conserved non-autonomous Helitron has recently invaded the genomes of multiple herbivorous insect species, and may have resulted from HTT vectored by viral particles. Re-annotation of the spontaneous vsk-1d1 Autographa californica nucleopolyhedrovirus insertion element $[27,28]$ as a non-autonomous Helitron with high similarity to TEs in Trichoplusia $n i$ and Spodoptera frugiperda cell lines provided putative evidence for contemporary HTT of this insect-derived Helitron. These data suggest that Helitrons may be capable of crossing taxonomic boundaries and that HTT might be a potent force in shaping genome evolution.

\section{Results and discussion}

\section{Prediction and annotation of highly conserved Helitron} integrations

The transposition of novel mobile genetic elements into a genome affects not only the genetic structure and function, but can influence the evolutionary trajectories of a species. High degrees of DNA sequence similarity among copies of a TE in a genome are considered to be evidence of a recent invasion, wherein rapid transposition has not been subject to host repression and substitution mutations have not accumulated in the "young" propagates $[29,30]$. Additionally, evidence including unanticipated high sequence identity in TE families across the genomes of many taxa has been used to support the notion that mobile elements move across species boundaries $[17,26]$, where horizontal transposon transfer (HTT) is increasingly being viewed as a potent mechanism that influences genome evolution [31]. Indeed, the alignment of two previously annotated TEs, a miniature inverted repeat transposable element from Ostrinia nubilalis, OnMITE01 [32], and a Helitron element from B. mori, BmHel-2 [5], generated a 339 bp consensus that showed $66.2 \%$ sequence similarity and $77.55 \%$ similarity between $50 \mathrm{bp}$ at the $5^{\prime}$-end (Additional file 1) despite these nonprotein coding sequences being from species that have estimated divergence times of $>100$ mya [33]. Results from RepBase searches also indicated that a 149 bp region of OnMITE01 (positions 10 to 159) showed 79.2\% similarity (hit score $=619$ ) to nucleotides 973 to 827 of the $984 \mathrm{bp}$ Heliconius melpomene element Helitron-like-4a_Hmel [34]. This was not unanticipated since this Helitron was previously shown to share sequence similarity across lepidopteran genomes for elements in the assembled genomes of B. mori, Danus plexippus, H. melpomene, and Manduca sexta [5], and also to retain $\geq 74.4 \%$ homology between the genomes of $O$. nubilalis and multiple lepidopteran species including B. mori [32]. Due to the predicted co-ancestry of BmHel-2 and OnMITE01, and to adapt a common nomenclature for orthologous copies of the same element, 
the OnMITE01 element is from hereon referred to as OnHel-2.

Despite the prior prediction of homology among this Helitron family, the mode of propagation across the lepidopteran lineage has not been investigated. A broad homology-based search of contemporary DNA sequence resources identified 488, 735, 661, and 73 homologous Helitrons respectively in GenBank nr, dbEST, dbGSS, HTGS, and TSA database accessions (Additional file 2). DNA sequence identities $\geq 65.7 \%$ were observed to the OnHel-2 query among accession from species of insects from the Orders Lepidoptera $(n=24)$, Hymenoptera (2), Coleoptera (2), Hemiptera (1), and Phasmida (1), as well as sequences from insect bracovirus and baculovirus (5), and plant species (5). In many instances full-length elements could not be identified from GenBank accessions due to the short nature of many entries and is a limitation of many dbGSS or dbEST accessions. The inability to define full-length Helitrons also likely resulted when one or both termini remained cryptic, which was presumably due to mutations caused by the integration of other TEs or substitution mutation that obscured 5 '-TC and 3 '-CTRR motifs. Alternatively, sequence gain has been observed among Helitrons from maize [11,12] as well as Lepidoptera $[5,13]$ such that potential acquisition of large sequences may have negatively affected the detection of Helitron termini, but this was not investigated further in this study. Regardless, Helitron-like 5' -TC and $3{ }^{\prime}$-CTRR termini and flanking AT dinucleotides [1], and 3 '-stem-loops (hairpins) were predicted upstream of the $3^{\prime}$-CTRR termini of this Helitron family (Figure 1). Additional secondary structure elements were predicted for representative OnHel-2 and BmHel-2 Helitrons including a 5 '-inverted repeat (IR), subterminal inverted repeats (SIRs) that were originally described from drosopholid [4] and lepidopteran Helitrons [13,16]. Hel-2 copies from insects were distinctive in that a variablelength tetranucleotide repeat microsatellite $(\mathrm{CTGT})_{\mathrm{n}}$ was present downstream of the $5^{\prime}$-SIR. These results suggest that Hel-2-like Helitrons are a prevalent TE lineage in the genomes of Lepidoptera, and are analogous to other Helitrons from Lepidoptera in that they show broad distributions and share extensive sequence homology among copies integrated in the genomes of different species $[5,13,16]$.

\section{Horizontal transfer of Hel-2 Helitrons among insects and viruses}

TEs enter a genome lineage either through vertical inheritance from a progenitor species or by HTT. The "patchy distribution" of a TE among genomes of a taxa is typically used to support the historical occurrence for HTT. Helitron copy numbers were predicted to vary $\sim 33$-fold between the genomes of $H$. melpomene and $D$. plexippus [5], which suggests that TEs have had variable success at colonizing the genomes of Lepidoptera, or species with low observed Helitron densities have only recently experienced invasion by this TE. The variation in Helitron copy number between species is likely subject to stochastic processes of genetic drift when the TE initially invades a genome $[35,36]$, whereby newly acquired TEs can be lost or retained at low copy if transposition is not robust or germline transformation does not occur. Selection may also be a major constraint on TE propagation in a genome, where an equilibrium develops between TE gain and loss [37,38]. Due to potential loss of a TE from previously invaded genomes, evidence consisting of a "patchy distribution" across species of a taxonomic group may, in general, not be reliable evidence in support of HTT. Furthermore, sparse

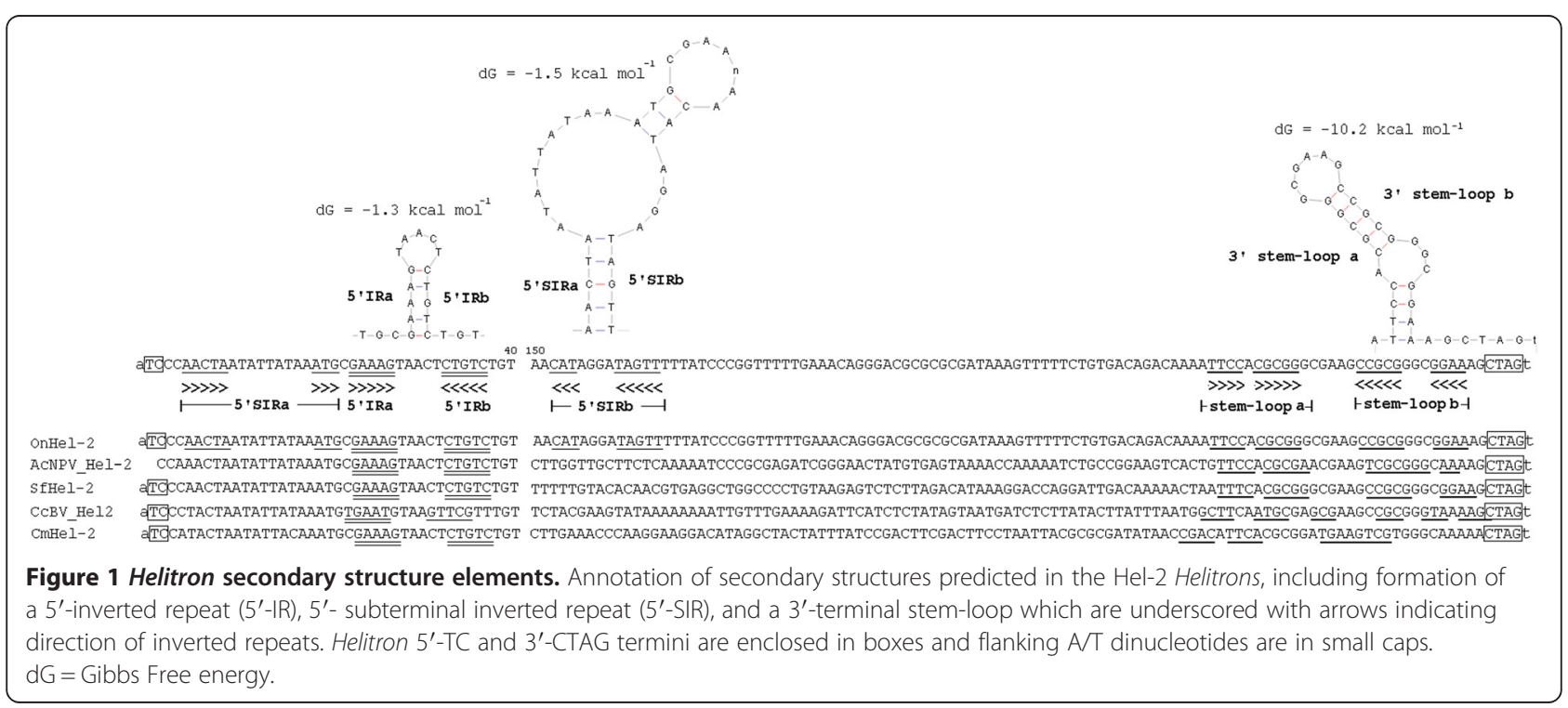


sequence resources for many species of Lepidoptera result in unreliable and inappropriate estimates of Helitron presence or absence using a bioinformatic approach, but may improve as additional genome resources become available [39].

When vertical movement of DNA descends to progenitor genomes at the point of complete speciation, the subsequent bifurcating relationships between $\mathrm{TE}$ and species phylogenies tend to be highly correlated. In contrast, genome invasion by HTT violates these correlations due to the crossing of species boundaries, wherein rates of TE divergence no longer parallel genome divergence of the hosts [40]. HTT essentially causes introgression of TE sequences across affected species and results in incomplete lineage sorting that tends to be problematic for phylogenetic algorithms. Indeed, phylogenetic reconstruction of the Hel-2 Helitron resulted in few well supported nodes (bootstrap values $\geq 50 \%$ ), but generally did reflect some of the broad relationships among species of Lepidoptera [33] in that Hel-2 copies in the genus Heliconius in the Superfamily Papilionidea (true butterflies) are well supported (Figure 2). The phylogenetic tree also showed distinct clades for Hel-2 copies from the species Plutella xylostella, Heliothis virescens, and Choristoneura fumiferana, which may be a consequence of more recent invasions of these genomes where Hel-2 copies are relatively homogenized due to insufficient time for substitution mutations to have accumulated. In contrast, two well supported clades with bootstrap values $\geq 52$ containing Hel- 2 copies from the genomes of multiple species from the Family Noctuidae (Figure 2) may indicate that Hel-2 copies are more closely related between noctuid species compared to within a species. This is highlighted by instances where Hel-2 Helitrons from the genome of Helicoverpa armigera show higher interspecific similarity and cluster with Hel-2 copies in other noctuid species including $T$. $n i$, and might provide partial evidence for HTT. Lower nucleotide divergence was predicted between these $H$. armigera and $T$. $n i$ Hel-2 Helitrons (Additional file 3A) compared to RpS5 housekeeping gene sequences (Additional file 3B; Table 1). Albeit narrow in scope, this suggests a more recent ancestry among TEs compared to the genomic background of these insect species, and in combination with phylogenetic analysis provide putative evidence of HTT. The inability to completely resolve this phylogeny was not surprising due to the short DNA sequence data inherent to nonautonomous Helitrons, which compared to analogous reconstructions using derived amino acid, are more prone to the effects of forward and back mutation or convergence over longer evolutionary time frames. Additionally, HTT is expected to cause incomplete lineage sorting which causes known problems with phylogentic analyses [41]. Thus, the construction of phylogenies from TE sequence data may become increasingly confounded with increasing evolutionary time and increasing incidence of HTT.

It is becoming generally accepted that TEs are transferred among eukaryotes with taxonomic close relationships, such as among genomes of insect [42], plant [43] and mammalian species [44], or among species that share pathogenic, parasitic or endosymbiont vectors $[17,21]$. Comparative genomic approaches have become feasible through the accumulation of increasing amounts of genome sequence data and provide additional, and arguably more reliable, evidence for detecting the occurrence and possible modes of HTT. Prior sequence homology evidence showed that a mariner-like element had recently been transferred between the genomes of an insect host, Adoxophyes honmai (Lepidoptera: Tortricidae), and it's parasitoid wasp, Ascogaster reticulatus (Hymenoptera: Braconidae), which suggested the "intimate" relationship between these species facilitated HTT by an unknown mechanism [45]. Additionally comparative genomic evidence documented the transposition and integration of a reptilian short interspersed nuclear element (SINE) into the genome of a dsDNA taterapox virus (TATV), which suggested that viruses are capable of acquiring host TEs and could potentially be a vector for HTT [46]. Two pervasive Helitrons from species of Lepidoptera were reported to have integrated into the genomes of dsDNA polydnaviruses (PDVs), the Cotesia plutella bracovirus (CpBV) and the C. sesamiae bracovirus (CsMBV) [17]. Analogously, results from this study detected novel Hel-2 elements integrated in the genomes of CpBV, CsMBV as well as $C$. congregata $\mathrm{BV}(\mathrm{CcBV})$ and $C$. vestalis $\mathrm{BV}$ (CvBV; Figure 3; Additional file 4) which were novel and not described in prior studies [17]. Similar to copies in the genomes of lepidopteran insects, Helitron-like termini and $(\mathrm{CTGT})_{\mathrm{n}}$ or $(\mathrm{GTTT})_{\mathrm{n}}$ repeats were also annotated in most viral and parasitoid wasp derived elements (Additional file 5). This accumulating evidence suggests that the biology and mode of PDV infection may facilitate HTT among related Lepidoptera. Specifically, the genomes of PDVs are segmented and integrated into the genomes of their primary hosts (parasitoid wasps; Hymenopteran: Ichneumonidae and Braconidae), but these viral genome segments also exist as free circular dsDNAs that become enclosed into transmissible viral particles $[47,48]$. PDV virons are produced in specialized ovarian cells of the parasitoid wasps and are transmitted by female wasps during oviposition into larval Lepidoptera. Infecting PDVs act to suppress the immune response of lepidopteran larvae, and thus have evolved a mutual relationship with the wasps [49]. PDV DNA enters the nucleus of infected cells wherein viral gene products may downregulate larval immune response genes and could potentially be targeted for integration by actively transposing host Helitrons. Our data analysis suggest that a Hel-2 


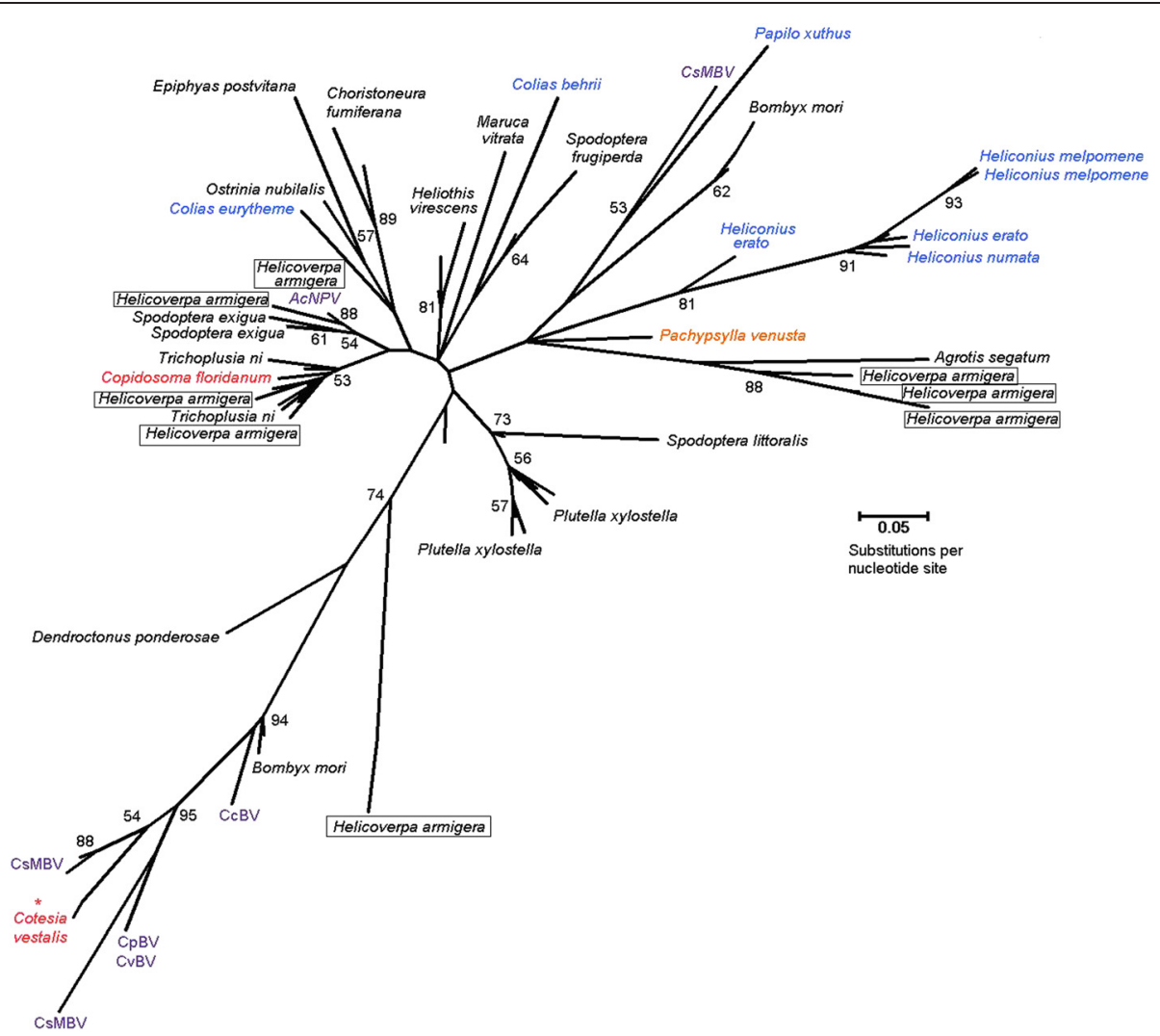

Figure 2 Phylogenetic relationship among Helitron-like Hel-2 elements horizontally transferred among insects and viruses. Maximum likelihood (ML) estimation among Hel-2 elements in genomes of moths, butterflies, hymenopteran parasitoids, a hemipteran gall former, and insect viruses. using the Tamura-3-Parameter model and Gamma shape parameter of 2.249. Bootstrap support is indicated at each node of the consensus tree when values were $\geq 50 \%$, whereas unresolved branches exist for all other instances. Resulting tree has a Log Likelihood $=-1908.7$; total branch length of 5.438. Species definitions: Moths: Family Bombycidae, Bombyx mori; Family Crambidae, Ostrinia nubilalis and Maruca vitrata; Family Noctuidae, Agrotis sgatum, Helicoverapa armigera, Heliothis virescens, Spodoptera frugiperda, S. exigua, and S. littoralis; Family Tortricidae, Choristoneura fumiferana and Epiphyas postvittana, Family Plutellidae, Plutella xylostella Butterflies: Family Pieridae, Colias eurytheme and C. behrri, Family Papilionidae, Papilio xuthus; Family Nymphalidae, Heliconius erato, H. numata and $\mathrm{H}$. melpomene. ${ }^{*} \mathrm{C}$. vestalis TSA submissions determined to share flanking sequence with CvBV segements and are suggested to comprise transcripts derived from viral integrations.

element integrated into the genome of the parasitoid chalcid wasp, Copidosoma floridanum, also shows a close phylogenetic relationship with Hel-2 elements in the genome of its primary host, T. ni [50] (Figure 2). This is suggestive but not conclusive evidence that HTT may occur through tri-tropic associations between parasitoids wasps, PDVs and host Lepidoptera, where HTT of another Helitron between T. ni and C. floridanum lacked evidence of PDV involvement [27].

In addition to the detection of Hel-2 integrations in PDVs, databases search results from the current study show that a mutant form of Autographa californica nucleopolyhedrovirus (AcNPV) contained a Hel-2 Helitron integration (Additional file 2). AcNPV particles directly infect larval

Table 1 Comparative divergence between Helitron and host genomic sequence

\begin{tabular}{|c|c|c|c|c|c|c|c|}
\hline GenBank accession & Species & 1. & 2. & 3. & 4. & 5. & 6. \\
\hline JX683313.1 & 1. O. nubilalis & 100.00 & 79.01 & 76.77 & 75.45 & 83.11 & 67.77 \\
\hline JN383812.1 & 2. P. xylostella & 81.66 & 100.00 & 78.45 & 75.33 & 80.09 & 73.63 \\
\hline AY837869.1 & 3. T. ni & 85.21 & 85.89 & 100.00 & 88.62 & 78.47 & 74.02 \\
\hline KF624964.1 & 4. H. armigera & 85.89 & 83.60 & 86.60 & 100.00 & 77.46 & 75.90 \\
\hline FE273699.1 & 5. C. fumiferana & 82.27 & 84.22 & 82.09 & 83.16 & 100.00 & 63.84 \\
\hline AY769319.1 & 6. B. mori & 82.39 & 80.60 & 83.98 & 82.19 & 79.43 & 100.00 \\
\hline
\end{tabular}

Matrix showing nucleotide identity between Helitrons (above diagonal) and genomic sequences (ribosomal protein $\mathrm{S} 5$; below diagonal). Instances of higher similarity between TEs compared to host genomes are bolded. Sequences used in comparisons are provided in Additional file 3. 


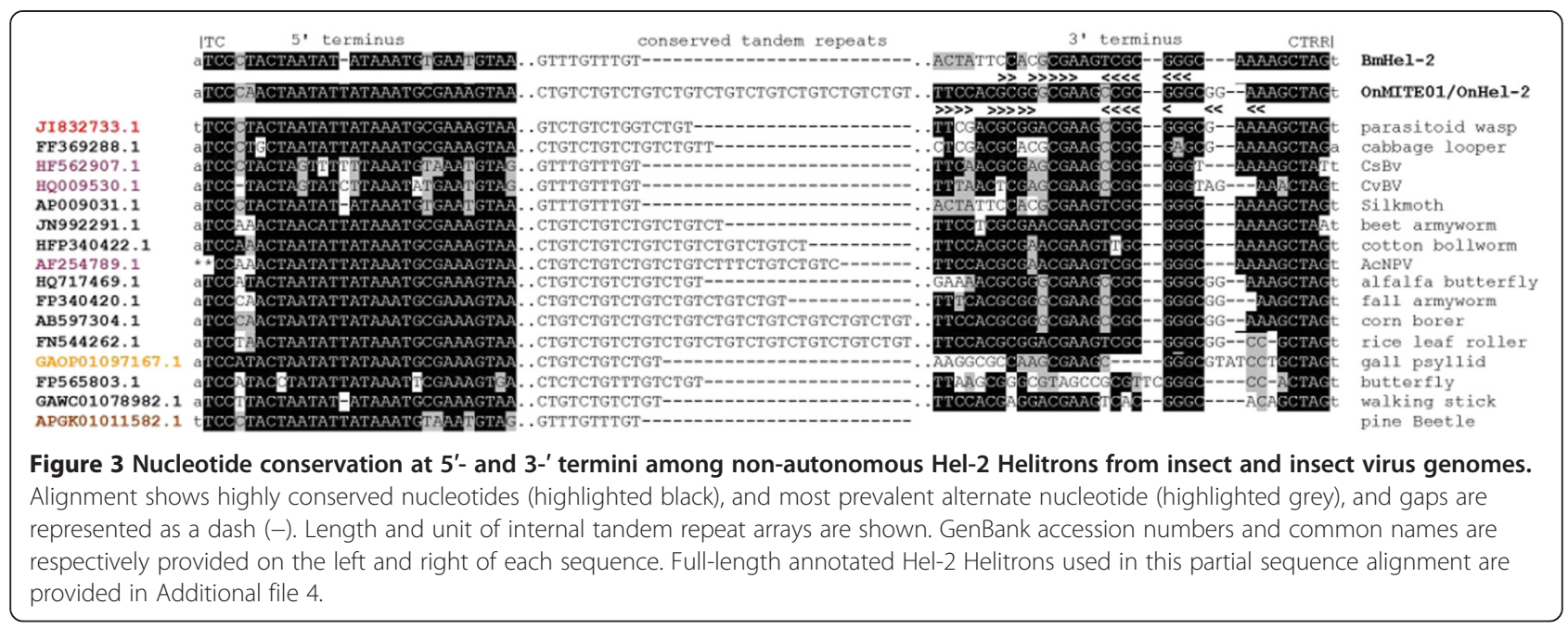

Lepidoptera including the Alfalfa Looper, Autographa californica (Lepidoptera: Noctuidae) and other species in the lepidopteran family Noctuidae. This previously described integration encompassed a 1006 bp regions of the AcNPV mutant vsk-1d1 that was reported to have spontaneously inserted into the PstI- 1 region between ORFs 28 and 29 of the AcNPV genome after serial passage through $T . n i$ and S. frugiperda cell lines $[28,51]$. Our analysis showed that the 1006-bp AcNPV genome insertion showed 5'-TCC and 3'-CTAG terminal Helitron motifs, a predicted 3'-stem-loop as well as $\geq 83.2 \%$ sequence identity with Hel-2 Helitrons from T. $n i$ and S. frugiperda (Figure 1). More detailed analysis of the 1006-bp Hel-2 from AcNPV suggests that the mobile element may be comprised of $5^{\prime}$ - and $3^{\prime}$ - Hel-2 termini interrupted by an approximately 577-bp region with homology to a $S$. frugiperda transposon named TELNCR [52] which accounts for increased size compared to other Hel-2 elements. Moreover, the termini of the AcNPV Hel-2 element show 95.63 and 95.37\% nucleotide identity to Helicoverpa zea and $H$. armigera Hel-2 elements (Figure 4). Given that these two insect integrated elements shared $97.58 \%$ nucleotide identity, might indicate that this viral insertion element was likely derived from an insect genome. Even though portions were annotated using genome data from different insect species, this likely does not necessarily suggest that the AcNPV element has acquired this chimeric structure as a result of HTT but could also be result of the current inadequacy of genome sequence data. Although prior instances have documented the transposition of lepidopteran TEs into viruses $[17,53,54]$, integration of Hel-2 into AcNPV after serial passage through permissive hosts provides a rare instance where HTT was observed in real time. In addition to this direct evidence of Hel-2 integration into AcNPV, the phylogenetic relationships of PDV and AcNPV Helitrons are intertwined with Hel-2 elements from insects. Specifically, strong bootstrap support was obtained for nodes defining close relationships between Hel-2 Helitrons from

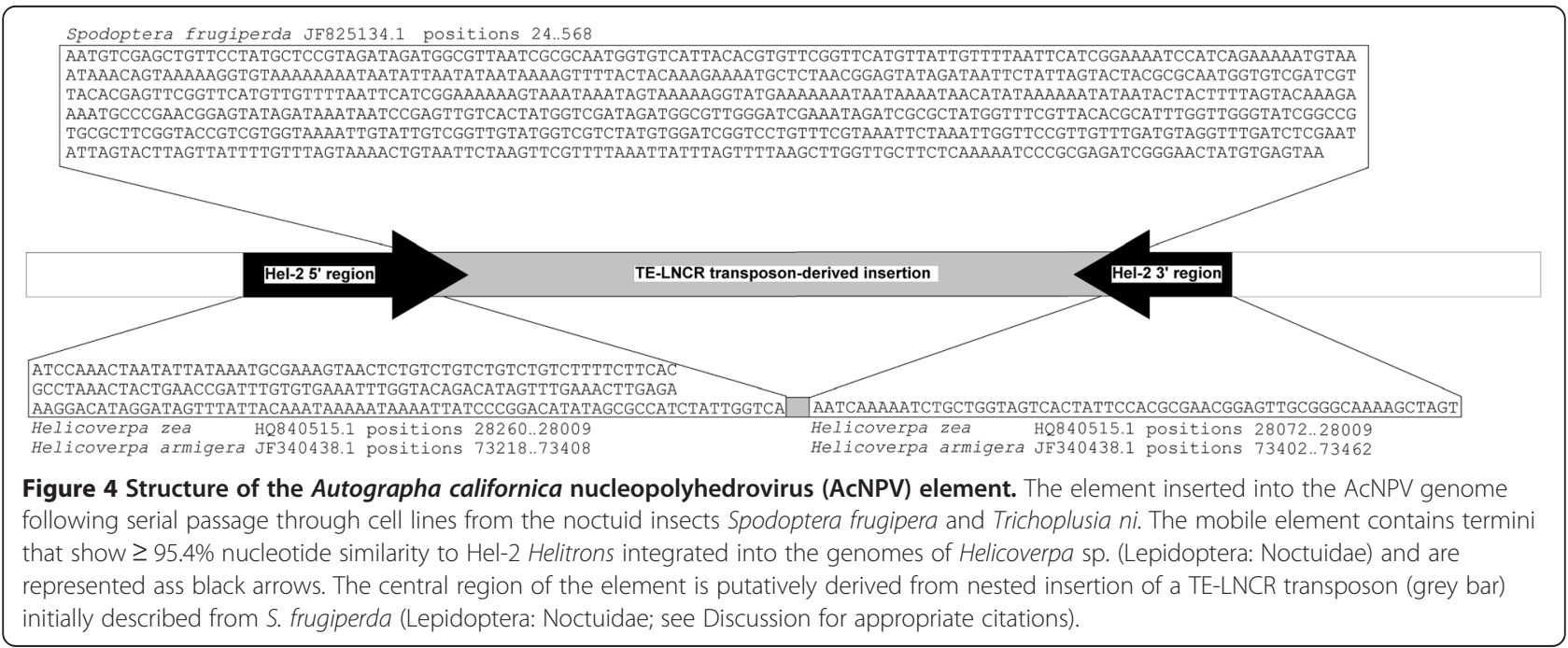


AcNPV and Helicoverpa armigera and Spodoptera exigua (Figure 2), and the Hel-2 element in AcNPV is comparatively more distant from those integrated into PDVs. Similarly, Helitrons from PDVs, and the lepidopteran species B. mori, Cotesia vestalis, and Papilo xuthus formed a wellsupported cluster. It should be noted that Hel-2 copies identified in TSA accessions from the wasp C. vestalis shared strong sequence identity upstream of the $5^{\prime}$-TC and downstream of the $3^{\prime}$-CTAG termini with the PDV, $\mathrm{CvBV}$, which was interpreted to indicate that the sequence were actually derived from an infecting virus (data not shown). In contrast, sequence flanking the Hel-2 copy identified from a TSA entry from the parasitoid wasp Copidosoma floridanum (Hymenoptera: Encyrtidae) show no homology to known virus DNAs, although origin from yet undescribed viruses cannot be ruled out. In conjunction with prior research outlined above, the comparative genomic data in this study indicate that viral genomes likely have acquired mobile elements from their insect hosts, with the assumption that cross-infection of related species by the virus and subsequent re-mobilization of the TE can result in HTT.

\section{Conclusions}

Only recently has HTT been shown to occur between more distantly related taxa, where unknown mechanisms resulting from close associations between organisms have been implicated. For example, TEs from the DNA transposon hAT superfamily were shown to retain high levels of sequence homology among Chordates, Arthropods, Mollusks, and Platyhelminths, and provided estimates of genome invasion times that predated speciation as evidence for HTT [26]. Transfer of hAT transposons appeared to occur when host-parasite interactions present opportunities for exchange of cellular and genetic material, such as between the blood feeding insect Rodnius prolixus and mammals, or the pond snail Lymnaea stagnalis and vertebrates [26]. Both of these interactions involve an intermediate vector; the trypanosome Trypanosoma cruzi vectored by $R$. prolixus, and parasidic flatworms (trematodes) that are transmitted by $L$. stagnalis. This vectordependent mechanism of HTT may be considered analogous to the viral-mediated horizontal transfer of Helitrons among Lepidoptera detected here and previously [17]. These data also suggest that HTT can occur across species of distant evolutionary relationships, and that the resulting spread of mobile elements into naive species may dramatically reshape genome structure and function [30]. Many further investigations as well as evidence of HTT of other TEs are definitely warranted before mechanisms or vectoring agents can be proposed.

Data presented here suggests that genetic information can be exchanged among highly diverged species. Although viruses were previously shown to vector Helitrons among insects and parasites have been implicated in the movement of TEs among tetrapods, evidence presented here may be the initial annotation of real-time HTT between insect and insect viral genomes. Although the mechanisms remain unclear and shear number of insect-derived TE integrations predicted in parasite genomes appears infrequent, the consequences of this movement may be substantial given the ancestral relationships between insects and their associated viruses. This suggests that HTT mediated by insectpathogen interactions might affect the genome evolution of the species involved through mobile DNA transfer.

\section{Methods}

\section{Prediction and annotation of highly conserved Helitron integrations}

Previously identified TEs, the BmHel-2 from the genome of the silkmoth, Bombyx mori [5] and a 239 bp miniature inverted repeat transposable element (MITE01) characterized in O. nubilalis BAC inserts [32] (nucleotide positions 29 to 273 of GenBank accession ET217030.1) comprise highly similar DNA elements. The DNA sequence similarity among MITE01 and BmHel-2 sequences were estimated by alignment between $O$. nubilalis from GenBank accessions AB597304.1 (positions 1429 to 1179) and EF396411.1 (685 to 943), and B. mori accessions AP009003.1 (89798 to 90133) and AP009031.1 (54931 to 54604) using the ClustalW algorithm (Gap penalty = 10; Gap Extension - 0.2). BmHel-2 contains sequence features that led to classification as a Helitron-like TE [5], but OnMITE01 was previously described as a putative miniature inverted repeat transposable element (MITE) [9]. Additional annotation of MITE01 included prediction of putative primary sequence and secondary structure features consistent with other Helitron-like TEs from Lepidoptera using methods described previously $[13,16]$ using the Mfold DNA server [55] (http://mfold.rna.albany.edu/?q=mfold/dna-folding-form).

Novelty of MITE01 and BmHel-2 TEs was investigated by using respective sequences as queries against all sequence sources in RepBase (http://www.girinst.org/censor/index. php) [56].

A sequence homology approach was used to predict Helitron integrations in publically available genome sequence resources, and relied upon sequence conservation among non-autonomous Helitron-like TEs among Lepidoptera $[13,16,17,32]$. In this instance, the MITE01 and BmHel-2 Helitron sequences were used as queries to search the non-redundant (nr) nucleotide database $(\mathrm{nr})$, expressed sequence tag (dbEST), genome survey sequence (dbGSS), high throughput genome sequence (HTGS), and transcriptome sequence assembly (TSA) databases housed at the National Center for Biotechnology Information (NCBI). Query of whole genome sequences from $B$. mori, D. plexipus, $H$. melponene, and M. sexta were previously conducted for BmHel-2 [5], 
and were not repeated in this study. All web-based queries used the blastn algorithm parameters adjusted for divergent sequences (discontinuous blastn) [57], and database search results were filtered as described by Coates et al. [13]. Results were further filtered for those with homology (65\% sequence identity and $E$-values $\leq 10^{-5}$ ) to the entire query length, or modified to $\geq 50 \mathrm{bp}$ to either the $5^{\prime}$ - or $3^{\prime}$ - ends to capture partial sequence data or copies that have undergone insertion mutations caused by other TEs.

\section{Horizontal transfer of Hel-2 Helitrons among insects and viruses}

Incongruence between established interspecies relationships ("species trees") and TE phylogenies can provide evidence for the HT of elements among genomes. A consensus alignment among $77 \mathrm{Hel}-2$ elements indentified from searches of GenBank nr, dbEST, dbGSS, HTGS and TSA databases was constructed using the ClustalW algorithm in the MEGA 5.2.1 alignment Suite [58] using default parameters, except the gap penalty was set to 10.0. Manual adjustments were made to the multiple sequence alignment, and converted to .meg format. The MEGA 5.2.1 software package [58] was further used to determine the optimal model of sequence evolution and gamma shape parameter. A Maximum Likelihood (ML) tree was constructed using the Tamura-3-parameter model with completed deletion of gapped data, and a consensus tree was reported from among random trees constructed from 1000 bootstrapped pseudoreplicates of the aligned sequence data [59] using MEGA 5.2.1 software package [58] (branch support $\geq 50 \%$ reported). Incomplete lineage sorting, convergence or other phenomenon may likely influence the resulting largely unresolved Hel-2 phylogeny, such that evolutionary relationships among HTT events could not be ascertained. Species relationships among Families of Lepidoptera were obtained from published estimates [33], and used to make ad hoc comparisons to the obtained Hel-2 phylogeny for qualitative description of distributions of lepidopteran species in well-supported TE clades.

\section{Additional files}

Additional file 1: Figure S1. Estimation of sequence similarity between Hel-2 Helitrons from Ostrinia nubilalis (previously named OnMITE01; Coates et al. [31]) and Bombyx mori (Han et al. [5]) identified from GenBank accessions. A) Alignment of Hel-2 Helitron sequences from Bombyx mori and Ostrinia nubilalis using the CLUSTAL 2.1 algorithm. Flanking a/t dinucleotides from host genomic DNA are in small caps, Helitron-like $5^{\prime}$-TC and $3^{\prime}$-CTAG termini are underlined, and bases involved in formation of the $3^{\prime}$-stem-loop are indicated with arrows. Homologous nucleotides positions are highlighted and gaps are indicated as dashes (-). B) Percent identity matrix between B. mori and O. nubilalis Hel-2 Helitrons.
Additional file 2: Table S1. Table of BLASTn search results and associated GenBank nr database accessions with sequence similarity to Hel-2 Helitron queries.

Additional file 3: Data S1. Fasta formatted data used for estimation of sequence divergence between genomic housekeeping genes and Hel-2 elements among Lepidoptera.

Additional file 4: File S3. GenBank accessions used in Figure 1 of the main text annotated with Hel-2 sequence features of a $5^{\prime}-\mathrm{TC}$ and $3^{\prime}-\mathrm{CTAG}$, and $(C T G T)_{n}$ or $(G T T T)_{n}$ microsatellite repeats are indicated. Flanking genomic DNA is in small caps.

Additional file 5: Figure S2. Full GenBank sequence accessions containing Hel-2 Helitrons integrations in the genomes of A) lepidopteran infecting viruses, and B) parasitoid wasps. Helitron-like 5'-TC and 3'-CTAG, and $(\mathrm{CTGT})_{\mathrm{n}}$ or $(\mathrm{GTTT})_{\mathrm{n}}$ microsatellite repeats are indicated. Flanking genomic DNA is in small caps.

\section{Abbreviations}

HTT: Horizontal transposon transfer; DNA: Deoxyribonucleic acid; TE: Transposable element; MITE: Miniature inverted repeat transposable element; RCR: Rolling circle replication; ssDNA: Single stranded deoxyribonucleic acid; RPA: Replication protein A; mya: Million years ago; nr: Non-redundant; dbEST: Database of expressed sequence tags; dbGSS: Database of genome survey sequences; HTGS: High throughput genome sequence; TSA: Transcriptome sequence assembly; SIR: Subterminal inverted repeat; RpS: Ribosomal protein small subunit; SINE: Short interspersed nuclear element; CpBV: Cotesia plutella bracovirus; CsMBV: Cotesia sesamiae bracovirus; CcBV: Cotesia congregate bracovirus; CsBV: Cotesia vestalis bracovirus; PDV: PolyDNA virus; dsDNA: Doublestranded DNA; AcNPV: Autographa californica nucleopolyhedrovirus.

\section{Competing interests}

The author declares that he has no competing interests.

\section{Authors' contributions}

BC applied all bioinformatic methods, analyzed and interpreted data, and wrote the paper.

\section{Acknowledgements}

This research was a joint contribution from the United States Department of Agriculture (USDA), Agricultural Research Service (ARS) (CRIS Project 3625-22000-017-00), and the lowa Agriculture and Home Economics Experiment Station, lowa State University, Ames, IA (Project 3543). Funding was by USDA-ARS Congressional Appropriated funds, NP304 Crop Protection and Quarantine, Project Tile: Ecologically-based Management of Insect Pests of Corn, with Emphasis on Corn Borers, Rootworms, and Cutworms. This article reports the results of research only. Mention of a proprietary product does not constitute an endorsement or a recommendation by USDA for its use. USDA is an equal opportunity employer and provider.

Received: 26 June 2014 Accepted: 3 February 2015

Published online: 27 February 2015

\section{References}

1. Kapaitonov W, Jurka J. Rolling-circle transposons in eukaryotes. Proc Natl Acad Sci U S A. 2001;98:8714-9.

2. Lal SK, Giroux MJ, Brendel B, Vallejos CE, Hannah LC. The maize genome contains a Helitron insertion. Plant Cell. 2003;15:381-91.

3. Pritham EJ, Feschotte C. Massive amplification of rolling-circle transposons in the lineage of the bat Myotis lucifugus. Proc Natl Acad Sci U S A. 2007;104:1895-900.

4. Yang HP, Barbash DA. Abundant and species-specific DINE-1 transposable elements in 12 Drosophila genomes. Genome Biol. 2008;9:R39.

5. Han MJ, Sen YH, Xu MS, Liang HY, Zhang HH, Zang Z. Identification and evolution of the silkworm Helitrons and their contribution to transcripts. DNA Res. 2013;20:471-84.

6. Liu H, Fu Y, Li B, Yu Z, Zie J, Cheng J, et al. Widespread horizontal gene transfer from circular single-stranded DNA viruses to Eurkaryotic genomes. BMC Evol Biol. 2011;11:276. 
7. Gallivotti A, Zhao Q, Kyozuka J, Meeley RB, Ritter MK, Doebley JF, et al. The role of barren stalk1 in the architecture of maize. Nature. 2004;432:630-5.

8. Choi JD1, Hoshino A, Park KI, Park IS. Spontaneous mutations caused by a Helitron transposon, Hel-lt1, in morning glory, Ipomoea tricolor. Plant J. 2007:49:924-34.

9. Inagaki $\mathrm{S}$, Nakamura K, Morikami A. A link among DNA replication, recombination, and gene expression revealed by genetic and genomic analysis of TEBICHI gene of Arabidopsis thaliana. PLoS Genet. 2009;5:e1000613.

10. Morgante M, Brunner S, Pea G, Fengler K, Zuccolo A, Rafalski A. Gene duplication and exon shuffling by helitron-like transposons generate intraspecies diversity in maize. Nature Genet. 2005;37:997-1002.

11. Barbaglia AM, Klusman KM, Higgins J, Shaw JR, Hannah LC, Lal SK. Gene capture by Helitron transposons reshuffles the transcriptome of maize. Genetics. 2012;190:965-75.

12. Lai J, Li Y, Messing J, Dooner HK. Gene movement by Helitron transposons contributes to haplotype variability of maize. Proc Natl Acad Sci U S A. 2005;102:9068-73.

13. Coates BS, Hellmich RL, Grant DM, Abel CA. Mobilizing the genome of Lepidoptera through novel sequence gains and end creation by non-autonomous Lep1 Helitrons. DNA Res. 2012;19:11-21.

14. Galagan JE, Calvo SE, Cuomo C, Ma LJ, Wortman JR, Batzoglou S, et al. Sequencing of Aspergillus nidulans and comparative analysis with $A$. fumigatus and A. oryzæ. Nature. 2005;438:1105-15.

15. Ray DA, Feschotte C, Pagan HJT, Smith JD, Pritham EJ, Arensburger P, et al Multiple waves of recent DNA transposon activity in the bat, Myotis lucifugus. Genome Res. 2008;18:717-28.

16. Coates BS, Sumerford DV, Hellmich RL, Lewis LC. A Helitron-like transposon superfamily from Lepidoptera disrupts $(G A A A)_{n}$ microsatellites and is responsible for flanking sequence similarity within a microsatellite family. J Mol Evol. 2010;70:278-88.

17. Thomas J, Schaack S, Pritham EJ. Pervasive horizontal transfer of rolling-circle transposons among animals. Genome Biol Evol. 2010;2:656-64.

18. Thomas J, Vadnagara K, Pritham EJ. DINE-1, the highest copy number repeats in Drosophila melanogaster are non-autonomous endonuclease-encoding rolling-circle transposable elements (Helentrons). Mob DNA. 2014;5:18.

19. Silva JC, Kidwell MG. Horizontal transfer and selection in the evolution of P elements. Mol Biol Evol. 2000;17:1542-57.

20. Coates BS, Hellmich RL, Lewis LC. Nuclear small subunit rRNA group I intron variation among Beauveria spp provide tools for strain identification and evidence of horizontal transfer. Curr Genet. 2002:41:414-24.

21. Vavre F, Fleury F, Lepetit D, Fouillet P, Bouletreau M. Phylogenetic evidence for horizontal transmission of Wolbachia in host-parasitoid associations. Mol Biol Evol. 1999;12:1711-23.

22. Klasson L, Kambris Z, Cook PE, Walker T, Sinkins SP. Horizontal gene transfer between Wolbachia and the mosquito Aedes aegypti. BMC Genomics. 2009;10:33.

23. Waterhouse DF, Norris KR. Biological control - pacific prospects. Melbourne: Inkata Press; 1987

24. Polaszek A, Walker AK. The Cotesia flavipes species complex: parasitoids of cereal stem borers in the tropics. Redia. 1991;74:335-41.

25. Houch MA, Clark JB, Peterson KR, Kidwell MG. Possible horizontal transfer of Drosophila genes by the mite Proctolaelaps regalis. Science. 1991;253:1125-9.

26. Zhang HH, Feschotte C, Han MJ, Zhang Z. Recurrent horizontal transfers of Chapaev transposons in diverse invertebrate and vertebrate animals. Genome Biol Evol. 2014;6:1375-86.

27. Guo X, Gao J, Li F, Wang J. Evidence of horizontal transfer of non-autonomous Lep1 Helitrons facilitated by host-parasite interactions. In: Scientific reports. 2014. p. 4.

28. Kumar S, Miller LK. Effects of serial passage of Autographa californica nuclear polyhedrosis virus in cell culture. Virus Res. 1987;7:335-49.

29. Gilbert C, Schaack S, Pace II JK, Brindley PJ, Feschotte C. A role of host-parasite interactions in the horizontal transfer of transpososons across phyla. Nature. 2010;464:1347-50.

30. Brookfield JF, Badge RM. Population genetics models of transposable elements. Genetica. 1997;100:281-94.

31. Yang HP, TI H, You TL, Yang TH. Genome-wide comparative analysis of the highly abundant transposable element DINE-1 suggests a recent transpositional burst in Drosophila yakuba. Genetics. 2006;173:189-96.

32. Schaack S, Gilbert C, Feschotte C. Promiscuous DNA: horizontal transfer of transposable elements and why it matters for eukaryotic evolution. Trends Ecol Evol. 2010;25:537-46.
33. Coates BS, Sumerford DV, Hellmich RL, Lewis LC. Repetitive genomic elements in a European corn borer, Ostrinia nubilalis, bacterial artificial chromosome library were indicated by bacterial artificial chromosome end sequencing and development of sequence tag site markers: implications for lepidopteran genomic research. Genome. 2009;52:57-67.

34. Wahlberg $\mathrm{N}$, Wheat CW, Peña C. Timing and Patterns in the taxonomic diversification of Lepidoptera (Butterflies and Moths). PLoS One. 2013;8:e80875.

35. Lavoie CA, Platt RN, Novick PA, Counterman BA, Ray DA. Transposable element evolution in Heliconius suggests genome diversity within Lepidoptera. Mob DNA. 2013;4:1-10.

36. Brookfield JFY. Models of repression and transposition in PM hybrid dysgenesis by $P$ cytotype and by zygotically encoded repressor proteins. Genetics. 1991;128:471-86.

37. Charlesworth B, Lapid A, Canada D. The distribution of transposable elements within and between chromosomes in a population of Drosophila melanogaster. II. Inferences on the nature of selection against elements. Genet Res. 1992;60:115-30

38. Charlesworth B, Sniegowski P, Stephan W. The evolutionary dynamics of repetitive DNA in eukaryotes. Nature. 1994;371:215-20.

39. i5K Consortium. The i5K Initiative. Advancing arthropod genomics for knowledge, human health, agriculture, and the environment. J Heredity. 2013;104:595-600.

40. Daniels SB, Peterson KR, Strausbaugh LD, Kidwell MG, Chovnick A. Evidence for horizontal transmission of the $\mathrm{P}$ transposable element between Drosophila species. Genetics. 1990;124:339-55.

41. Maddison WP, Knowles LL. Inferring phylogeny despite incomplete lineage sorting. Syst Biol. 2005;55:21-30.

42. Robertson HM, Lampe DJ. Recent horizontal transfer of a mariner transposable element among and between Diptera and Neuroptera. Mol Biol Evol. 1995;12:850-62.

43. Diao $X$, Freeling $M$, Lisch D. Horizontal transfer of a plant transposon. PLoS Biol. 2005;4:e5.

44. Pace JK, Gilbert C, Clark MS, Feschotte C. Repeated horizontal tranfer of a DNA transposon in mammals and other tetrapods. Proc Natl Acad Sci U S A. 2008:105:17023-8.

45. Yoshiyama M, Tu Z, Kainoh Y, Honda H, Shono T, Kimura K. Possible horizontal transfer of a transposable element from host to parasitoid. Mol Biol Evol. 2001;18:1952-8

46. Piskurek O, Okada N. Poxviruses as possible vectors for horizontal transfer of retroposons from reptiles to mammals. Proc Natl Acad Sci U S A. 2007:104:12046-51

47. Stoltz DB, Krell PJ, Summers MD, Vinson SB. Polydnaviridae - a proposed family of insect viruses with segmented, double-stranded, circular DNA genomes. Intervirology. 1984;21:1-4.

48. Espagne E. Genome sequence of a polydnavirus: insights into symbiotic virus evolution. Science. 2004;306:286-9.

49. Strand MR. Polydnavirus gene products that interact with the host immune system. In: Beckage NE, Drezen JM, editors. Parasitoid viruses: symbionts and pathogens. Oxofrd: Elsevier; 2012. p. 149-61.

50. Strand MR. Development of the polyembryonic parasitoid Copidosoma floridanum in Trichoplusia ni. Entomol Exp et Appl. 1989;1:37-46.

51. McLachlin JR, Escobar JC, Harrelson JA, Clem RJ, Miller LK. Deletions in the Ac-iap1 gene of the baculovirus AcMNPV occur spontaneously during serial passage and confer a cell line-specific replication advantage. Virus Res. 2001:81:77-91.

52. Stanojcic S, Gimenez S, Permal E, Cousserans F, Quesneville H, Fournier P, et al. Correlation of LNCR rasiRNAs expression with heterochromatin formation during development of the holocentric insect Spodoptera frugiperda. PLoS One. 2011;6:e24746.

53. Jehle JA, Nickel A, Vlak JM, Backhaus H. Horizontal escape of the novel Tc1-like lepidopteran transposon TCp3.2 into Cydia pomonella granulovirus. J Mol Evol. 1998:46:215-24.

54. Friesen PD, Nissen MS. Gene organization and transcription of ted, a lepidopteran retrotransposon integrated within the baculovirus genome. Mol Cell Biol. 1990;10:3067-77.

55. Zuker M. Mfold web server for nucleic acid folding and hybridization prediction. Nucl Acids Res. 2003:31:3406-15.

56. Kohany O, Gentles AJ, Hankus L, Jurka J. Annotation, submission and screening of repetitive elements in Repbase: RepbaseSubmitter and Censor. BMC Bioinformatics. 2006;7:474 
57. Altschul SF, Gish W, Miller W, Myers EW, Lipman DJ. Basic local alignment search tool. J Mol Biol. 1990;215:403-10.

58. Tamura K, Peterson D, Peterson N, Stecher G, Nei M, Kumar S, et al. Molecular evolutionary genetics analysis using maximum likelihood, evolutionary distance, and maximum parsimony methods. Mol Biol Evol. 2011;28:2731-9.

59. Felsenstein J. Confidence limits on phylogenies: an approach using the bootstrap. Evolution. 1985;39:783-91.

Submit your next manuscript to BioMed Central and take full advantage of:

- Convenient online submission

- Thorough peer review

- No space constraints or color figure charges

- Immediate publication on acceptance

- Inclusion in PubMed, CAS, Scopus and Google Scholar

- Research which is freely available for redistribution 